\title{
Gerenciamento da Aprendizagem, Evasão em Ead Online e Possíveis Soluções: Um Estudo de Caso no IFCE
}

Gilvandenys Leite Sales - Instituto Federal do Ceará - denyssales@ifce.edu.br Eliana Alves Moreira Leite - Instituto Federal do Ceará - elimoreiraead@gmail.com Cassandra Ribeiro Joye - Instituto Federal do Ceará - cassandra@ifce.edu.br

Resumo. Este artigo trata dos possíveis impactos que modificações no gerenciamento da aprendizagem podem causar na evasão em EAD online. Adotou-se como metodologia um estudo de caso com grupos experimental e de controle constituídos por alunos do IFCE. Para o monitoramento das atividades do grupo de controle foi usada planilha eletrônica com lançamento manual de notas, as quais eram disponibilizadas para os alunos somente ao final da disciplina, enquanto ao grupo experimental foi associado um controle acadêmico automatizado com auxílio da ferramenta de avaliação formativa denominada Learning Vectors (LV). Com a análise dos dados obtidos percebeu-se que com o acompanhamento regulador e constante das atividades de aprendizagem por meio dos LV é possível inferir mudanças na taxa de evasão do IFCE.

Palavras-chaves: Avaliação formativa, Evasão, Learning Vectors, EaD online.

\section{Learning Management, Evasion in Online Distance Education and Possible Solutions: A Case Study in IFCE}

Abstract. This paper discusses the possible impacts that changes in management learning may have on evasion in distance education online. Methodology was adopted as a case study with experimental and control groups consisting of students IFCE. To monitor the activities of the control group was used spreadsheet with manual release notes, which were made available to students only at the end of the course, while the experimental group was associated with an academic control with the help of automated formative assessment tool called Learning Vectors (LV). With the data analysis it was realized that with the regulator and constant monitoring of learning activities through the LV is possible to infer changes in the dropout rate's IFCE.

Keywords: Formative Assessment, Evasion, Learning Vectors, e-Learning.

\section{Introdução}

A procura por qualificação, formação inicial ou capacitação continuada é cada vez maior na modalidade de Educação a Distância $(\mathrm{EaD})$, sendo a geração de EaD Online, aprendizagem com suportes digitais via rede web, a responsável pelo aumento da procura de cursos nessa modalidade, superando até mesmo a procura por cursos presenciais. Relativo a cursos de graduação na modalidade EaD, o Censo da Educação Superior (BRASIL, 2010), aponta um maior crescimento das matrículas em relação aos cursos presenciais no Brasil.

A princípio quem opta por esses cursos podem pensar nas vantagens que há em não precisar se locomover a um local presencial, na redução de custos que isso pode implicar e até mesmo numa falsa ideia que será mais fácil.

Dentre os desafios de quem oferta esses cursos, seja na modalidade semipresencial, presencial ou totalmente a distância, há de se destacar: a reformulação curricular, a produção adequada de conteúdos, a necessidade de mecanismos de 
gerenciamento e avaliação da aprendizagem, assim como a capacitação didáticopedagógica dos docentes nas diversas ferramentas disponibilizadas nos Ambientes Virtuais de Aprendizagem (AVA).

Paralelamente a essa euforia de crescimento há um fator que preocupa quem trabalha no gerenciamento desses cursos, a evasão.

O Relatório Analítico da Aprendizagem a Distância no Brasil (CensoEAD.br, 2010) mostra que o número de brasileiros que estudaram por meio da Educação a Distância em 2008 na educação superior perfaz um total de 786.718. A evasão média apresentada por esse censo, apurada entre as 129 instituições públicas e privadas participantes, foi de $18,5 \%$.

Mesmo que preocupante esse índice é menor quando comparado ao do ensino presencial. Nesse mesmo ano, segundo o Resumo Técnico do Censo da Educação Superior do INEP (BRASIL, 2009), o percentual de concluintes em relação aos ingressantes de 2005 na graduação presencial foi de 57,3\%, o que representa um total de $42,7 \%$ de evasão.

Relativo à evasão, uma questão que mais chama a atenção, segundo a pesquisa apresentada no Anuário Brasileiro Estatístico de Educação Aberta e a Distância (AbraEAD, 2008), "[...] é a clara constatação da evasão precoce. A quase totalidade dos alunos que deixam o curso o fazem logo no início, o que sugere mais atenção das instituições para esse relacionamento inicial com o estudante".

Esta mesma pesquisa elenca que os motivos mais citados que levam à evasão são "Financeiro", "Falta de tempo", "Não se adaptou ao método" e "Achou que o método EAD era mais fácil".

Esses dois últimos itens da pesquisa da AbraEAD (2008) tratam de uma questão de não adaptação à metodologia na modalidade não presencial. Segundo dados dessa pesquisa, a perspectiva dos alunos entrevistados, que se revelam decepcionados com $\mathrm{EaD}$, era que esse método seria diferente e, por isso, preferem o contato físico com professores e alunos.

Um dos fatores mencionados acima também é aceito por Nash (2005) apud Comarella (2009), o qual é a crença por parte dos estudantes, de que os cursos, por serem a distância, serão mais fáceis. Entretanto, para Nash (2005) é preciso gerenciar as expectativas desses estudantes, explanando no programa do curso claramente as atividades e os esforços necessários para concluí-lo.

Para Moore e Kearsley (2007), a evasão na EaD não se trata apenas de um fator isolado, mas de um conjunto de fatores que podem corroborar para o aluno desistir do seu curso. Para eles, alunos com clara intenção de concluir seu curso, que entregam suas tarefas antecipadamente ou que já concluíram outros cursos em $\mathrm{EaD}$, tem prognóstico de provável conclusão, porém alunos que não se enquadram nessa situação compõem um grupo de risco e merecem apoio adicional e aconselhamento.

Favero (2006) ressalta sobre a importância do diálogo para evitar a evasão do aluno na educação a distância. Nesse sentido, ele argumenta que uma das possíveis soluções que devem ser tomadas pelas universidades para dirimir a evasão deve ser "a de adotar tutores que fiquem sempre em contato com os alunos, na tentativa de que a atenção dispensada por aqueles não permita a evasão (p. 153)".

Ainda para tenta minimizar a evasão na $\mathrm{EaD}$ as instituições também têm se apoiado na tecnologia, nesse sentido Wilges et al. (2010) propõe a implementação da ferramenta AgentTool em ambientes virtuais de ensino e aprendizagem (AVEA), baseada no modelo de sistema multiagentes, de forma que haja um acompanhamento e monitoramento dos estudantes em todas as atividades que envolvem o ensino a V. $10 \mathrm{~N}^{\mathrm{o}} 3$, dezembro, 2012 
distância, para que assim possa avaliar até que ponto ferramentas dessa natureza podem contribuir para reduzir a evasão dentro das instituições de ensino na modalidade a distância.

Nesse contexto, este trabalho trata de um possível fator que pode ter contribuído para a redução da evasão nos cursos de graduação na modalidade em EaD do Instituto Federal do Ceará (IFCE), a avaliação formativa proporcionada pelo uso do Modelo Learning Vectors (LV). Implementado no ambiente virtual Moodle esse instrumento pode proporcionar um acompanhamento constante, regulação e suporte à aprendizagem, desde que a tutoria seja eficiente e presente.

Para tanto, na próxima seção relata-se sobre a avaliação em EAD e explica-se o Modelo LV de avaliação usado para monitoramento das atividades no ambiente virtual Moodle. Na seção 3 faz-se um estudo de caso comparando grupos de alunos nos quais foram aplicadas diferentes formas de gerenciamento da aprendizagem e a devida análise dos dados, por fim, na seção 4 tecem-se as considerações finais.

\section{A Avaliação em EaD}

Em EaD Online, as intervenções pedagógicas caracterizadas por feedbacks constantes às ações dos alunos devem ser estendidas a todos os momentos, inclusive quando se avalia. Segundo Perrenoud (1999), a avaliação deve ter o caráter de contínua formação: "É formativa toda a avaliação que ajuda o aluno a aprender e a se desenvolver, que participa da regulação das aprendizagens e do desenvolvimento no sentido de um projeto educativo".

Os Referenciais de Qualidade da Educação Superior a Distância no Brasil (BRASIL, 2007) acenam para que a avaliação do ensino e aprendizagem em EaD deva ser processual:

\footnotetext{
Na educação a distância, o modelo de avaliação da aprendizagem deve ajudar o aluno a desenvolver graus mais complexos de competências cognitivas, habilidades e atitudes, possibilitando-lhe alcançar os objetivos propostos. Para tanto, esta avaliação deve comportar um processo contínuo, para verificar constantemente o progresso dos alunos e estimulá-los a serem ativos na construção do conhecimento.
}

Esse mesmo documento ainda incita ao desenvolvimento de mecanismos de avaliação que possibilitem a contínua regulação na aprendizagem:

Desse modo, devem ser articulados mecanismos que promovam o permanente acompanhamento dos estudantes, no intuito de identificar eventuais dificuldades na aprendizagem e saná-las ainda durante o processo de ensino-aprendizagem (BRASIL, 2007).

Para fazer valer então uma avaliação formativa são necessárias metodologias, instrumentos apropriados, e mudanças comportamentais de quem acompanha o aluno nos Ambientes Virtuais de Aprendizagem (AVA), o Professor/Tutor, que precisa deixar de: priorizar notas e os resultados finais, ignorar os percursos individuais e reforçar o modelo tradicional de avaliação.

A não mudança na postura do Professor/Tutor pode gerar: abandono virtual, desmotivação e até mesmo a desistência do aprendiz de um curso em EaD online, o que implica em aumento dos índices de evasão.

Para Sales (2010):

Na sala de aula online, a avaliação como prática contínua deve promover a qualquer tempo as regulações nas aprendizagens. Para tanto, as ferramentas

V. $10 \mathrm{~N}^{\mathrm{o}} 3$, dezembro, 2012 
síncronas ou assíncronas do AVA devem favorecer as ações interventivas e formativas por parte do Professor/Tutor, que interagindo com cada aluno, ou grupos de alunos, proporciona-lhes condições propícias à aprendizagem.

Na subseção a seguir, trata-se de um modelo não-linear aplicado em EaD online, capaz de proporcionar uma avaliação processual e formativa: O Modelo Learning Vectors.

\subsection{O Modelo Learning Vectors (LV)}

O modelo de avaliação Learning Vectors (LV) (SALES, 2010) é fundamentado na mediação iconográfica e intervenções geradas pelo Professor/Tutor como forma de comunicação com seus alunos, o que pode ajudar a transformar o AVA em um ambiente dialógico e motivador ao permitir maior proximidade no acompanhamento das atividades propostas e o devido tratamento para problemas de aprendizagem ao longo do processo de formação, além de contribuir para suprir a sensação de solidão virtual, que a distância física impõe.

O modelo LV atua sobre as interações intermediadas pelas ferramentas síncronas e assíncronas do AVA. Os LV também armazenam as notas das atividades presenciais, que uma vez configuradas devem ser devidamente lançadas no mapa de notas LV, que também gerencia a frequência do aluno em fóruns, tarefas, wikis e chats, automação que se constitui em diferencial na redução da carga de trabalho.

O constante e contínuo desempenho e rendimento do aluno é feito pelo mecanismo denominado Vetor-Aprendizagem (Figura 1).

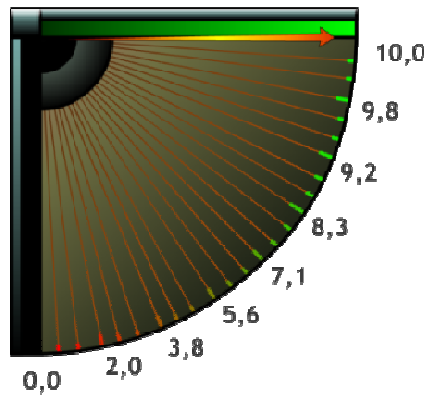

Figura 1 - Vetor-Aprendizagem

Uma escala de menções qualitativas associada a uma escala iconográfica, LV Ícones, é utilizada pelo Professor/Tutor para avaliar as mensagens ou arquivos enviados pelos alunos (Tabela 1) durante as atividades no AVA.

Tabela 1 - Menções qualitativas e LV Ícones

\begin{tabular}{l|llllll}
$\begin{array}{l}\text { Menções } \\
\text { Qualitativas }\end{array}$ & Muito Bom & Bom & Regular & Fraco & Não Satisfatório Neutro \\
\hline LV Ícones & (..) & (.) & (.) & (-)
\end{tabular}

Os LV ainda trazem uma referência ao nível de desempenho do aluno no curso. Expresso pelo Fator $\beta$, essa métrica envolve os resultados positivos e negativos das contribuições dos alunos nas atividades colaborativas ou individualmente desenvolvidas.

Para Sales, Barroso e Soares (2012): 
O Fator $\beta$ é um indicador qualitativo não-linear para suporte no acompanhamento da aprendizagem do aluno (...) Como métrica a ser tomada como padrão pedagógico de desempenho de qualidade da aprendizagem em $\mathrm{EaD}$ online, ora construída de forma partilhada na interação, ora construída por percursos individualizados, o Fator $\beta$ relaciona e associa os aspectos subjetivos tomados a partir dos itens de apreciação da Escala de Menções e transforma-os numa escala formal de avaliação definida por valores que exprimem a positividade em relação à negatividade do desempenho do aluno.

O desenvolvimento desse modelo ocorreu a partir da extensão e reuso de códigos das ferramentas do próprio AVA Moodle (MOODLE, 2012), que é um software livre muito bem documentado, modularizado e desenvolvido em uma das mais populares linguagens de programação para criação de aplicativos web, o PHP.

Por essa razão, as novas instâncias: Fórum LV, Tarefa LV, Wiki LV e Chat LV, bem como o controle de frequência puderam ser facilmente integradas e adaptadas a esse ambiente.

Na próxima seção, faz-se um estudo de caso comparando grupos nos quais foram aplicadas diferentes formas de gerenciamento da aprendizagem.

\section{Evasão: Um Estudo de Caso}

Nesse estudo de caso, cada grupo analisado - Grupo de Controle e Grupo Experimental - foi constituído por turmas que pertenciam a diferentes cidades polos do estado do Ceará, matriculadas no $1^{\circ}$ e $2^{\circ}$ semestres dos cursos de Hotelaria e Licenciatura em Matemática sob a coordenação da UAB/IFCE. A mesma proposta pedagógica foi aplicada em ambos os grupos, com os mesmos professores e materiais didáticos.

O objetivo foi observar nos grupos a possível influência sobre a taxa média de evasão que possa ter decorrido a partir da introdução do Modelo LV nas turmas, tendo em vista ter sido esta a única mudança significativa no processo, bem como verificar se, em uma sala de aula virtual, há dependência em relação às condições iniciais, e assim inferir que ela pertence a um sistema dinâmico complexo.

O número de alunos por turma a cada semestre foi computado diretamente das planilhas de notas, ou mapas de notas LV dos arquivos dos Professores/Tutores, significando, portanto, alunos que realmente iniciaram as disciplinas no semestre e não que apenas se matricularam no controle acadêmico da instituição.

Nessa análise, o Grupo de Controle é constituído por oito turmas, cujo acompanhamento da aprendizagem não foi feita com os LV. Na Tabela 2, apresentamse os dados compilados do $1^{\circ}$ e $2^{\circ}$ semestres e a taxa de evasão para esse grupo.

Tabela 2 - Evasão de alunos do Grupo de Controle sem a utilização dos LV

\begin{tabular}{|c|ccc|} 
& $\mathbf{1}^{\mathbf{0}}$ Semestre & $\mathbf{2}^{\mathbf{0}}$ Semestre & Evasão\% \\
Turma A & 30 & 20 & 33,3 \\
Turma B & 60 & 44 & 26,7 \\
Turma C & 50 & 27 & 46,0 \\
Turma D & 50 & 34 & 32,0 \\
Turma E & 50 & 29 & 42,0 \\
Turma F & 50 & 32 & 36,0 \\
Turma G & 47 & 34 & 27,7 \\
Turma H & 59 & 49 & 17,0 \\
\hline
\end{tabular}

Fonte: Dados da UAB/IFCE: A - Ubajara; B - Quixeramobim I; C - Meruoca; D - Limoeiro I; E Jurema; F - Caucaia; G - Limoeiro II; H - Quixeramobim II 
6

Para o controle de notas dessas turmas, e devido acompanhamento da aprendizagem, não se fez uso do mecanismo "Notas" do Moodle, uma vez que se desejava observar a dinâmica de interação entre alunos/alunos e alunos/Tutores, não somente em fóruns, mas também em chats e wikis, ferramentas estas não avaliadas pelo Moodle, mas cobertas pelos LV.

Para tanto, utilizou-se de planilha eletrônica com lançamento totalmente manual de notas pelo Professor/Tutor. O preenchimento dessas planilhas, que podiam conter até 60 alunos, tomava muito tempo do Professor/Tutor, visto que o preenchimento manual ocorria após a análise de toda informação gerada no curso e nos diversos relatórios oferecidos pelo Moodle, aluno por aluno. Isso dificultava ao aluno o acompanhamento de sua aprendizagem e acesso às suas avaliações, que só poderiam ser visualizadas caso o Professor/Tutor disponibilizasse as notas parciais.

O Grupo Experimental teve as mesmas condições iniciais de seleção, mas com um número menor de oferta de vagas, passou pelas mesmas disciplinas no $1^{\circ}$ semestre, a entrada acadêmica é defasada de um semestre em relação ao Grupo de Controle. Essa turma, primeira a utilizar os LV, experimentou sua metodologia de acompanhamento por meio dos Vetores-Aprendizagem em todas as disciplinas do $1^{\circ}$ semestre. É importante frisar que nessas turmas era a primeira vez que Professores/Tutores exerciam a ação de avaliar fazendo uso de todas as funcionalidades dos LV.

$\mathrm{Na}$ Tabela 3, apresentam-se os dados compilados do $1^{\circ}$ e $2^{\circ}$ semestres e a taxa de evasão para esse grupo.

Tabela 3 - Evasão de alunos do Grupo Experimental com a introdução dos LV

\begin{tabular}{l|ccc} 
& $\mathbf{1}^{\mathbf{0}}$ Semestre & $\mathbf{2}^{\mathbf{0}}$ Semestre & Evasão \\
Turma $\mathbf{A}_{\mathbf{L V}}$ & 35 & 27 & 22,9 \\
Turma $\mathbf{B}_{\mathbf{L V}}$ & 37 & 29 & 21,6 \\
Turma $\mathbf{C}_{\mathbf{L V}}$ & 24 & 14 & 41,7 \\
Turma $\mathbf{D}_{\mathbf{L V}}$ & 35 & 24 & 31,4 \\
Turma $\mathbf{E}_{\mathbf{L V}}$ & 35 & 31 & 11,4 \\
Turma $\mathbf{F}_{\mathbf{L V}}$ & 35 & 29 & 17,1 \\
Turma $\mathbf{G}_{\mathbf{L V}}$ & 35 & 26 & 25,7 \\
Turma $\mathbf{H}_{\mathbf{L V}}$ & 38 & 26 & 31,6 \\
\hline
\end{tabular}

Fonte: Dados da UAB/IFCE: A - Ubajara; B - Quixeramobim I; C - Meruoca; D - Limoeiro I; E Jurema; F - Caucaia; G - Limoeiro II; H - Quixeramobim II

Na Figura 2 apresenta-se um comparativo entre as taxas médias de evasão dos Grupos de Controle e Experimental, com exceção da Turma H do Grupo Experimental1. Observações diretas dos dados já permitem que se perceba uma redução nessa taxa.

\footnotetext{
${ }^{1}$ Investigando essa turma diretamente no polo do município de Quixeramobim/CE, colheram-se informações acerca do aumento da taxa de evasão, destaca-se a opção dos alunos por outros cursos superiores que já cursavam em outra instituição, sendo essa a principal causa.

V. $10 \mathrm{~N}^{\circ} 3$, dezembro, 2012
} 


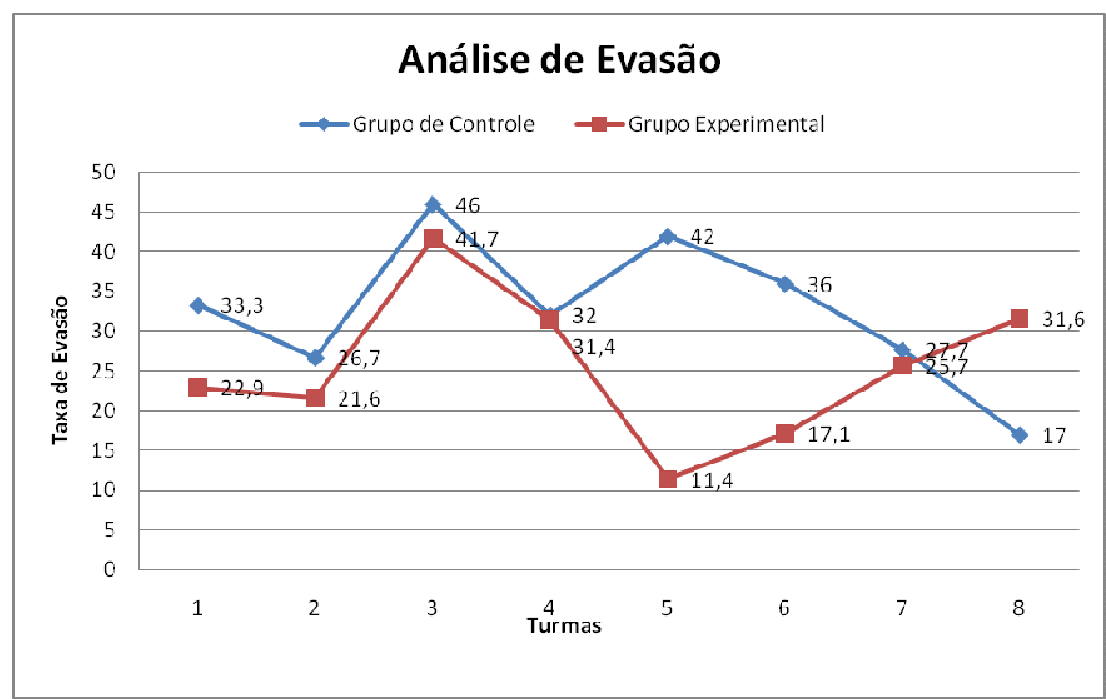

Figura 2 - Índices de evasão de alunos do $1^{\circ}$ para o $2^{\circ}$ semestre dos Grupos de Controle e Experimental

Os dados das Tabelas 2 e 3 são apresentados com suas médias $\left(\mathbf{X}_{\mathbf{L}}^{-} \mathbf{1}\right)$, desvios $\left(\mathrm{X}_{\mathrm{i}}-\overline{\mathrm{X}}\right)$, desvios quadráticos $\left(\mathrm{X}_{\mathbf{i}}-\overline{\mathbf{x}}\right)^{2}$, variâncias $(\mathrm{Si})$ e desvios padrões $(\sigma)$ na Tabela 4.

Tabela 4 - Tratamento estatístico dos dados de taxa média de evasão dos grupos analisados

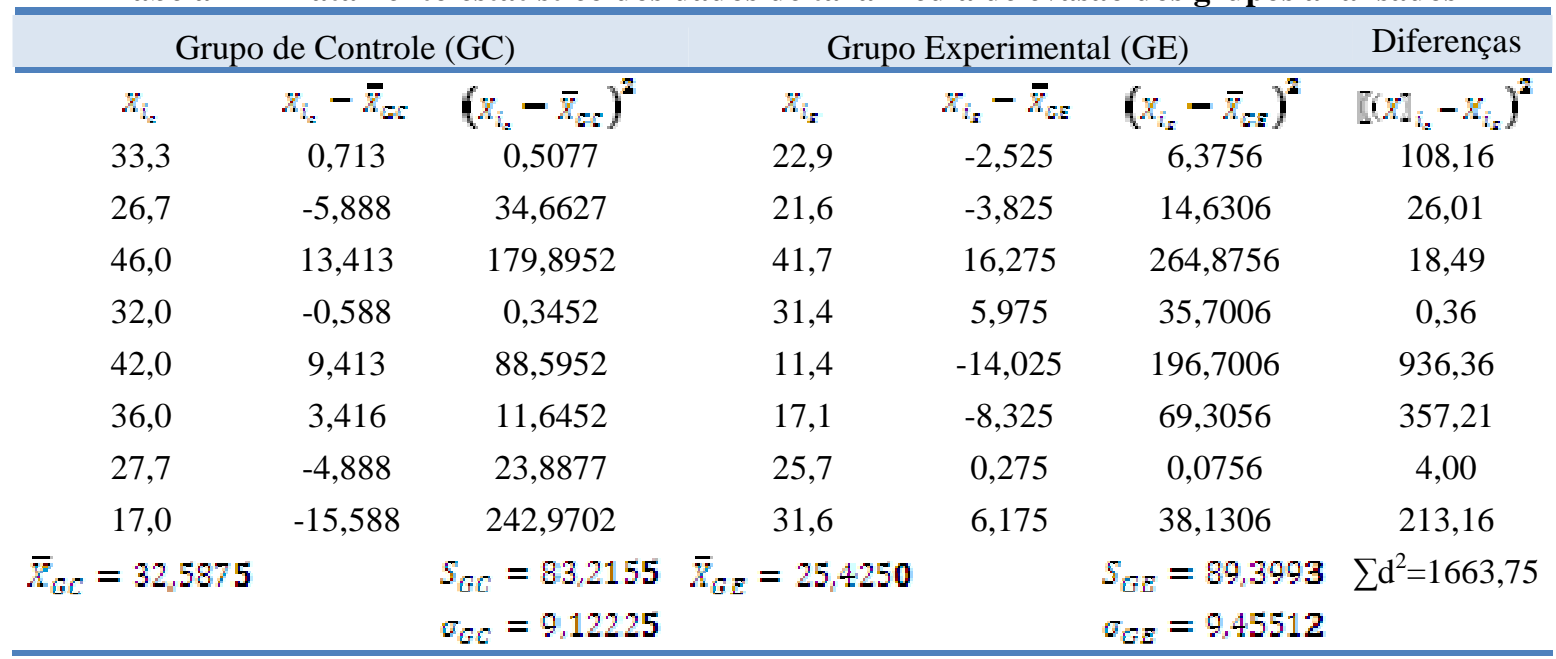

Para saber se é significativa a diferença entre as médias apresentadas na Tabela 4, aplicou-se um teste de significância estatística, mais especificamente um teste de diferenças entre médias populacionais.

O teste escolhido foi o Teste T, que aplica a distribuição t-Student, que é uma distribuição de probabilidade estatística. O Teste $\mathrm{T}$ é um teste estatístico de hipótese para comparação de médias de dados pertencentes a uma pequena amostra. Esse teste permite saber se a hipótese inicial levantada, ou hipótese nula $\left(\mathrm{H}_{0}\right)$, será aceita ou se será rejeitada.

No presente estudo de caso, para a hipótese $\mathrm{H}_{0}$, toma-se que não há diferenças significativas nas taxas médias de evasão dos grupos $\left(\mathbf{H}_{0}=0\right)$, ou seja, essa diferença é nula, tal hipótese será criticada pela hipótese $\mathrm{H}_{1}$, que prevê diferenças significativas $\left(\mathrm{H}_{1} \neq 0\right)$.

Sendo aceita a hipótese $\mathrm{H}_{0}$, a diferença entre as taxas médias de evasão dos grupos não será estatisticamente confirmada.

V. $10 \mathrm{~N}^{\mathrm{o}} 3$, dezembro, 2012 
Por tratar-se de uma situação em que se quer comparar as médias de evasão em um mesmo local: antes e após a aplicação do Modelo LV de avaliação, ou seja, pares amostrais idênticos, exceto no que se refere ao tratamento recebido, os dados foram considerados emparelhados com as diferenças entre pares tomadas como independentes e seguindo uma distribuição normal.

A variável de teste com 7 graus de liberdade $(\mathrm{GL}=\mathrm{N}-1$, sendo $\mathrm{N}=8 \mathrm{o}$ tamanho de pares da amostra) tem seu valor crítico, estabelecido a um nível de significância $(\alpha)$ de 5\%, obtido numa tabela de Distribuição t de Student, por:

$\left|t_{G L, \frac{\alpha}{2}}\right|=\left|t_{7,0025}\right|=2,3646$

A região de aceitação de $\mathrm{H}_{0}$ é mostrada na Figura 3. Para valores de $\mathrm{t}$ menores do que $-2,3646$ ou maiores do que 2,3646, ou seja, para valores em módulo maiores do que 2,3646 rejeita-se a hipótese $\mathrm{H}_{0}$.

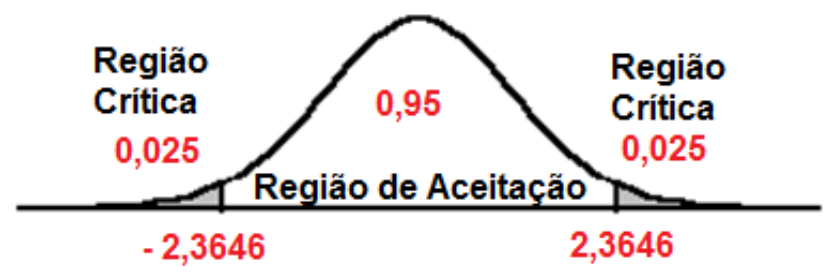

Figura 3 - Região provável de aceitação da hipótese nula $\mathbf{H}_{0}$

O desvio padrão das diferenças nas amostras $\left({ }^{\circ} \overline{\mathbb{d}}\right)$ é dado pela Equação 1:

$$
\sigma_{\bar{d}}=\sqrt{\frac{\sum d^{2}-N * \bar{d}^{2}}{N-1}}
$$

A variável de teste, ou valor da estatística do teste, é calculada pela Equação 2:

$t_{\text {Calculado }}=\sqrt{N} * \frac{\bar{d}}{\sigma_{\bar{d}}}$

Substituindo-se nas Equações 1 e 2 os dados obtidos na Tabela 5, com $\overline{\mathrm{d}}=\overline{\mathrm{X}}_{\mathrm{GC}}-\overline{\mathrm{X}}_{\mathrm{GE}}=7,1625$, encontram-se: $\sigma_{\mathrm{d}}=13,38090$ et $_{\text {Calculado }}=1,51399$.

Como o módulo da variável de teste calculado $\left(\mathrm{t}_{\text {Calculado }} \approx 1,5140\right)$ é menor que o módulo da variável de teste crítica $\left(\left|t_{7 ; 0,025}\right|=2,3646\right)$, obtido na tabela de Distribuição t de Student, a hipótese $\mathrm{H}_{0}$ é aceita a 5\% de significância.

Assim, de acordo com o resultado do Teste $\mathrm{T}$, nesta análise comparativa das taxas médias de evasão das turmas, pode-se afirmar com um nível de $95 \%$ de confiança, ou uma margem de erro de 5\%, que não existem diferenças significativas entre essas taxas médias nesse nível de confiabilidade.

Para confirmar esse resultado, uma análise e interpretação dos dados estatísticos e tratamento das informações, foram apuradas através do sistema SPSS (Statistical Package for the Social Sciences) (Figura 4). O SPSS é um software aplicativo que consiste num pacote estatístico para as ciências sociais e é aplicado em testes estatísticos de correlação, multicolinearidade e de hipóteses. 


\begin{tabular}{|c|c|c|c|c|c|}
\hline \multicolumn{6}{|c|}{ Paired Samples Statistics } \\
\hline & & Mean & $\mathrm{N}$ & Std. Deviation & $\begin{array}{l}\text { Std. Error } \\
\text { Mean }\end{array}$ \\
\hline \multirow[t]{2}{*}{ Pair 1 } & G.Controle & 32,5875 & 8 & 9,12225 & 3,22520 \\
\hline & G.Experimental & 25,4250 & 8 & 9,45512 & 3,34289 \\
\hline
\end{tabular}

Paired Samples Correlations
\begin{tabular}{|c|r|r|r|r|}
\hline & \multicolumn{1}{|c|}{$N$} & Correlation & \multicolumn{1}{c|}{ Sig. } \\
\hline Pair 1 & $\begin{array}{l}\text { G.Controle \& } G . \\
\text { Experimental }\end{array}$ & 8 &,- 037 &, 930 \\
\hline
\end{tabular}

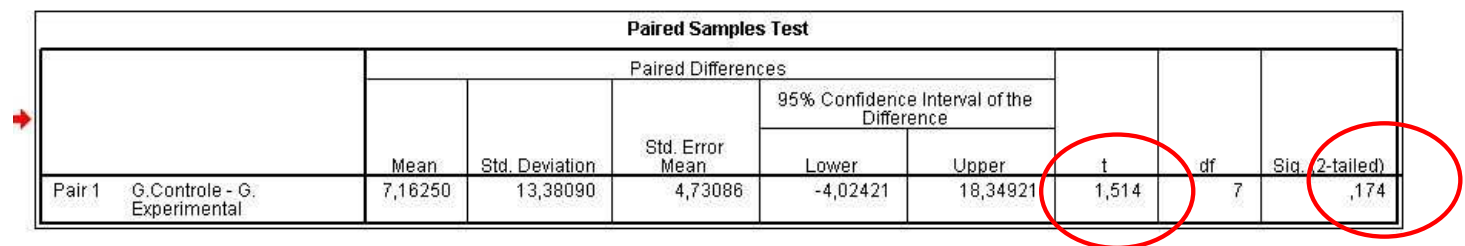

Figura 4 - Tratamento das informações das taxas médias de evasão apuradas através do sistema SPSS $(p$-value $=0,174)$

A interpretação dos dados dispostos na Figura 4 permite que se conclua que a diferença entre as taxas médias de evasão dos dois grupos, conforme a probabilidade pvalue $=0,174$, garante um grau de confiabilidade de $82,6 \%$.

Esse resultado, aparentemente não muito satisfatório estatisticamente, deve-se ao fato de a amostra ser pequena, apresentar dois pares de taxas médias com valores muito próximos, turmas 4 e 7, e ainda um par de valores, turma 8, todas da Figura 2, não seguindo a mesma regularidade dos demais pares que apresentam redução na taxa de evasão.

Ao fazer outra aplicação com o SPSS, dessa vez descartando o par de valores da taxa média de evasão da turma 8 , obtém-se o valor estatístico do teste, $\mathrm{t}=2,495$, que é maior que o módulo da variável de teste crítica, $\left|t_{7 ; 0,025}\right|=2,3646$, nesse caso, a hipótese $\mathrm{H}_{0}$ seria rejeitada a 5\% de significância. Podendo-se afirmar com um nível de 95\% de confiança que existem diferenças significativas entre essas taxas médias de evasão.

\section{Considerações Finais}

As análises realizadas sobre os dados coletados indicam que a introdução de um modelo de avaliação que proporcione mediação contínua e avaliação formativa pode influenciar nos índices de evasão.

Sabe-se que há outros fatores que levam à evasão em $\mathrm{EaD}$, mas no caso específico da presente pesquisa aplicada no IFCE, o fato de sua redução pode estar relacionada à introdução dos LV no Grupo Experimental, uma vez que seu emprego permitiu aos alunos um maior acompanhamento de suas aprendizagens e aos Professores/Tutores mais tempo para a mediação e intervenções necessárias, visto que ajudaram a reduzir o tempo de trabalho na análise e avaliação da carga de informações geradas.

Os LV automatizam as notas lançadas ao longo do curso para cada aluno, além de gerar relatórios de notas, gerenciar a frequência do aluno nas diversas ferramentas síncronas e assíncronas do AVA.

Pode-se também inferir que essa alteração na metodologia de avaliação de somativa para formativa, constituiu-se em mudança significativa das condições iniciais V. $10 \mathrm{~N}^{\mathrm{o}} 3$, dezembro, 2012 
na sala de aula virtual e pode ter contribuído para uma menor evasão de alunos no IFCE.

O acompanhamento das notas e Fator $\beta$, compiladas automaticamente no sistema ao longo do processo de aprendizagem, também permitiram melhores condições de gerenciamento das informações, conferindo ao Professor/Tutor mais tempo para as atividades de mediações e feedbacks aos alunos, assim como serviu de alerta quanto à evasão, auxiliando nas tomadas de decisões.

A presente pesquisa mostrou que a introdução de um modelo de avaliação formativa, modelo LV, contribuiu para a redução da evasão nos cursos de graduação na modalidade semipresencial do IFCE. Propõe-se para trabalhos futuros um estudo com uma maior quantidade de indivíduos e o levantamento do grau de satisfação dos usuários com a metodologia proposta.

\section{Referências bibliográficas}

AbraEAD. Anuário Brasileiro Estatístico de Educação Aberta e a Distância. Coordenação: Fábio Sanchez. São Paulo: Instituto Monitor, 2008.

Brasil (2007). Referenciais de Qualidade para Educação Superior a Distância Versão Preliminar. Ministério da Educação. Secretaria de Educação a Distância. Brasília, 2007.

Brasil (2009). Resumo Técnico do Censo da Educação Superior 2008. Ministério da Educação. Instituto Nacional de Estudos e Pesquisas Educacionais Anísio Teixeira. Brasília, 2009.

CensoEAD.br: Relatório Analítico da Aprendizagem a Distância no Brasil. Organização Associação Brasileira de Educação a distância. São Paulo: Pearson Education do Brasil, 2010.

Brasil. Censo da educação superior: 2010 - resumo técnico. - Brasília : InstitutoNacional de Estudos e Pesquisas Educacionais Anísio Teixeira, 2012. Disponível em: http://download.inep.gov.br/educacao_superior/censo_superior/resumo_tecnico/resumo tecnico_censo_educacao_superior_2010.pdf Acesso: 12/10/2012.

Comarella, R. L. Educação superior a distância: evasão discente. Dissertação de Mestrado. Programa de Pós-Graduação em Engenharia e Gestão do Conhecimento. Universidade Federal de Santa Catarina, Florianópolis. 2009.

Favero, R. V. M. Dialogar ou evadir: Eis a questão! Um estudo sobre a permanência e a evasão na educação a distância. Dissertação de Mestrado da Faculdade de Educação da Universidade Federal do Rio Grande do Sul, 2006.

Moodle. Disponível em: http://moodle.org/ Acesso: 12/10/2012.

Moore, M; Kearsley, G. A educação a distância: uma visão integrada. São Paulo: Thomson Learning, 2007. 
Perrenoud, P. Avaliação: da excelência à regulação das aprendizagens entre duas lógicas. Porto Alegre: Artes Médicas, 1999.

Sales, G. L. Learning Vectors (LV): um modelo de avaliação da aprendizagem em EaD online aplicando métricas não-lineares. Tese Doutorado. Departamento de Engenharia de Teleinformática. Universidade Federal do Ceará. 2010. $239 f$.

Sales, G.; Barroso, G.; Soares, J. Learning Vectors (LV): Um Modelo de Avaliação Processual com Mensuração Não-Linear da Aprendizagem em EaD online. In: Revista Brasileira de Informática na Educação, Volume 20, Número 1, 2012.

Wilges, B.; Ribas, J. C. da C; Catapan, A. H.; Bastos, R. C. Sistemas Multiagentes: mapeando a evasão na educação a distância. In: Revista Novas tecnologias na Educação, Volume 8, Número 1, 2010. 